\title{
Voriconazole and Posaconazole Improve Asthma Severity in Allergic Bronchopulmonary Aspergillosis and Severe Asthma with Fungal Sensitization
}

\author{
LiVingstone ChishimbA, B.SC., M.B.CH.B., M.R.C.P., ${ }^{1,2, *}$ ROBERT M. NiVEN, \\ B.SC., M.B.CH.B., F.R.C.P., M.D., M.FOM., ${ }^{1,2}$ JOHN COOLEY, M.SC., ${ }^{1}$ AND DAVID W. \\ DENNING, M.B.B.S., D.C.H., PH.D., M.R.C.P., F.MED.SCI. ${ }^{1,2}$ \\ ${ }^{I}$ Manchester Academic Health Science Centre, The University of Manchester, Manchester, UK. \\ ${ }^{2}$ The National Aspergillosis Centre, North West Lung Centre, University Hospital of South Manchester, Manchester, UK.

\begin{abstract}
Rationale and objectives. Severe asthma with fungal sensitization (SAFS) and allergic bronchopulmonary aspergillosis (ABPA) are progressive allergic fungal lung diseases whose effective treatment remains to be established. Current treatment with itraconazole is associated with a $40 \%$ failure rate and adverse events (AEs). We assessed the effect of voriconazole or posaconazole as second- and third-line therapies. Methods. We conducted a retrospective review of adult asthmatic patients with either ABPA or SAFS receiving voriconazole or posaconazole. Clinical, radiological, and immunological evaluation was used to assess response. Results. There were 25 patients, ABPA $(n=20)$ or SAFS $(n=5)$, 10 males, median age $=58$ years. All patients had failed itraconazole $(n=14)$ or developed AEs $(n=11)$. There were 33 courses of therapy analyzed, 24 with voriconazole and 9 with posaconazole. Clinical response to voriconazole was observed in 17/24 (70\%) patients at 3 months, 15/ $20(75 \%)$ at 6 months, and 12/16 (75\%) at 12 months compared with $7 / 9(78 \%)$ at 3, 6, and 12 months for posaconazole. Eighteen of $24(75 \%)$ patients discontinued oral corticosteroids (OCS), 12 of them within 3 months of therapy. Asthma severity was downgraded from severe to moderate $(n=8)$ and moderate to mild $(n=1)$ asthma in 9 of $24(38 \%)$ asthmatic patients. There was a marked reduction in OCS and short-acting beta-2 agonist use, health-care utilization due to asthma, and improvement in overall health status. Furthermore, there was a statistically significant reduction in immunological markers appearing at 9 months $(p=.008)$ for total IgE and at 12 months for radioallergosorbent test IgE for Aspergillus fumigatus $(p=.0056)$. Six of $23(26 \%)$ patients on voriconazole had AEs requiring discontinuation before 6 months compared with none on posaconazole $(p=.15)$. Four relapsed (57\%), one at 3 months and three at 12 months after discontinuation. Conclusion. Both voriconazole and posaconazole are potentially effective alternative treatment options for SAFS and ABPA and may improve asthma control and reduce severity, though larger prospective studies are required to support these retrospective study findings.
\end{abstract}

Keywords allergic fungal pulmonary disease, Aspergillus, asthma control, fungal sensitization, severe asthma, treatment

\section{INTRODUCTION}

In recent years, there has been an increasing interest in the treatment options of allergic fungal airway diseases. Allergic bronchopulmonary aspergillosis (ABPA) and severe asthma with fungal sensitization (SAFS) are slowly progressive, debilitating allergic fungal diseases that affect the respiratory tract in asthma. The past decade has seen several studies of antifungal treatment improve patient quality of life (QOL), reduce corticosteroid requirements, and prevent lung damage.

The link between asthma severity and fungal allergy is strong (1-3). Indeed, sensitization to fungal allergens is associated with the risk of severe asthma exacerbations requiring multiple hospital and intensive care admissions $(4,5)$.

$\mathrm{ABPA}$ is the most frequently recognized manifestation of allergic aspergillosis, with three published case series estimating that $0.7-3.5 \%$ of the referred asthma population has ABPA $(6,7)$. It is characterized by exaggerated Th2 CD4+ allergic inflammatory response to Aspergillus fumigatus

*Corresponding author: Livingstone Chishimba, Respiratory Research, The University of Manchester, 2nd Floor Education \& Research Centre, Wythenshawe Hospital, Southmoor Road, Manchester M23 9LT, UK; Tel: +44 161291 5811; Fax: +44 161291 5806; E-mail: Livingstone. Chishimba@manchester.ac.uk allergens in the bronchial airway of atopic asthma and Cystic fibrosis (CF) patients when A. fumigatus spores are inhaled and germinate in bronchial mucus releasing allergens including proteases.

The clinical and pathological course of ABPA (8) is variable, including recurrent exacerbations or chronic persistent symptoms. The disease may remit temporarily but is more commonly a progressive, unremitting disorder, and untreated patients have a chronic course characterized by recurrent pulmonary consolidation and in many cases progression to bronchiectasis or pulmonary fibrosis (8), bronchiolitis obliterans (9), granulomatous bronchiolitis (9), lung destruction (10), chronic cavitary aspergillosis (CPA) (11), and, in rare cases, pleural effusion (12).

In recent years, a new asthma variant called "SAFS" has been described by Denning et al. (1). These patients have severe asthma and are sensitized to one or more fungi, but have normal or only slightly elevated total IgE concentrations (1). The clinical and diagnostic manifestations of SAFS are thought to arise from an allergic response to multiple antigens expressed by A. fumigatus and possibly other fungi, colonizing the bronchial mucus (13).

Traditionally, treatment for allergic fungal diseases involves the use of systemic oral corticosteroids (OCS) 
(14-19). Despite good efficacy, the chronic use of OCS has a number of adverse effects. In SAFS, even high-dose, prolonged OCS courses may be only moderately successful. Similarly, in some asthmatics, corticosteroids do not prevent the development of ABPA, and patients who develop the syndrome while on corticosteroids may have a protracted course with poor response to the usually effective doses of corticosteroids (16).

Since the introduction of itraconazole, the first oral antifungal agent with activity against Aspergillus species in 1991, some efficacy in ABPA has been documented (20-24). Randomized controlled trials show that treatment with oral itraconazole offers therapeutic benefit to approximately $60 \%$ of patients (25-27). A marked beneficial effect on QOL and other end points has been demonstrated among patients with SAFS treated with itraconazole $(27,28)$. A retrospective study by Pasqualotto et al. (27) in 2009 showed that itraconazole therapy for 6-12 months in both SAFS and ABPA is beneficial by reducing immunological markers (total IgE and specific IgE for A. fumigatus), eosinophil count $(p=.037)$, dose of oral steroids $(p=$ $.043)$, the number of courses of systemic steroids required by $57.4 \%(p=.041)$, and improvement in lung function (median FEV1 increased by $190 \mathrm{~mL} ; p$ $=.016$ ) at 6 months, although there were no statistically significant changes in these key variables at 12 months therapy compared with baseline.

However, a major problem with the use of itraconazole is the high failure rate and frequent adverse events (AEs) of around 39\% of cases treated (29). Such AEs include nausea and vomiting (approximately 10\%), diarrhea and flatulence $(2 \%)$, constipation, hyperlipidemia (1-9\%), hypokalemia (6\%), liver enzyme elevations $(2-7 \%)(29$, 30 ), peripheral edema (28), and peripheral neuropathy (31). Perhaps most importantly, adrenal suppression (44\%), especially when combined with some inhaled corticosteroids (ICS; 32), is recognized. Occasionally, itraconazole has been associated with heart failure (33). The optimal duration of therapy is unclear, given that only short-term (8-month) efficacy has been demonstrated for itraconazole (25), and there are unanswered questions about the safety of prolonged use of this therapy (29). In addition, reversible adrenal suppression with ICS and itraconazole in $50 \%$ of patients is a real concern (26). Furthermore, there is not yet any reliable evidence that this treatment ameliorates any progressive lung damage (25).

Recent developments have led to a renewed interest in finding more potent therapy. Some emerging data from studies of ABPA in cystic fibrosis indicate that voriconazole may be a useful adjunctive therapy for ABPA in $\mathrm{CF}$ $(34,35)$. Little is known about the response rates or appropriateness of alternative antifungal treatment for those with ABPA or SAFS. In this study, we assessed the effect of voriconazole or posaconazole as second- and third-line therapies in SAFS and ABPA in asthmatics attending the National Aspergillosis Centre.

\section{METHODS}

\section{Study Design and Setting}

We conducted a retrospective case-note review to assess the efficacy and safety of voriconazole or posaconazole. The study population (26 adults with ABPA $(n=21)$ or SAFS ( $n$ $=5)$ ) included all cases treated with either antifungal agent at the National Aspergillosis Centre, based at the University Hospital of South Manchester, UK. Information from case notes, laboratory results, and radiological investigation was entered into an ACCESS database. A detailed review of all chest radiological images was made through PACS (Picture Archiving and Communication System).

Inclusion Criteria. All patients had previously received itraconazole but had discontinued because of AEs (peripheral neuropathy $(n=2)$, heart failure $(n=1)$, hepatotoxicity $(n=3)$, profound adrenal suppression $(n=2)$, persistent nausea and vomiting $(n=5)$ or lack of clinical efficacy $(n=11)$, low serum concentrations $(n=1)$, or itraconazole resistance in A. fumigatus $(n=2))$.

The diagnostic criteria for $A B P A$ included (i) asthma (of any severity), (ii) total serum $\operatorname{IgE} \geq 1000 \mathrm{kU} / \mathrm{L}$, (iii) immediate cutaneous reaction to A. fumigatus of $>3 \mathrm{~mm}$ compared with control or (iv) elevated A. fumigatus-specific serum IgE levels, (v) precipitating antibodies to A. fumigatus in the serum, (vi) a history of pulmonary infiltrates (transient or fixed), (vii) central bronchiectasis (CB), (viii) a history of expectoration of brown plugs or flecks, and (ix) isolation of A. fumigatus from the sputum $(36,37)$, of which (ii) and (iv) were essential.

Severe asthma was defined according to the British Thoracic Society (BTS) criteria (treatment steps 4 or 5) (38). We used published criteria for the diagnosis of SAFS that included (i) severe asthma, (ii) total $\operatorname{IgE}<1000 \mathrm{kU} / \mathrm{L}$, and (iii) positive skin test or raised specific IgE to any fungus.

Treatment and Response. Voriconazole (300-600 mg/ day) or posaconazole $(800 \mathrm{mg} /$ day; adjusted by plasmalevel monitoring) was given for at least 6 months, if tolerated. The target plasma levels used were those used for invasive and chronic pulmonary aspergillosis, namely voriconazole pre-dose $1.3-5.7 \mathrm{mg} / \mathrm{L}$ (39) and posaconazole random $>0.7 \mathrm{mg} / \mathrm{L}$ after steady state was reached (40). Some patients received both agents at separate times, and each course is evaluated separately. Courses of therapy of at least 4 weeks were evaluated for efficacy $(n=34)$, as done previously in the FAST study (36). At least one spirometry measurement was done. We evaluated response in clinical, immunological, lung function, peripheral blood eosinophilia, or radiological features. Among these improvement parameters, clinical improvement was considered the most important parameter.

Clinical improvement was present if there was an improvement in symptoms and/or overall asthma control as defined by the GINA criteria that included reduction in dosage and courses of oral steroids (OCS), dosage of inhaled steroids (ICS), exacerbations, intercurrent infections, daytime or nocturnal symptoms, reduction in number of hospitalizations or emergency room visits for 
respiratory diseases, improvement in exercise tolerance (ET), lung function (FEV1), increase in energy, or improvement in overall QOL as defined by the patients' perception of symptoms. Symptoms of breathlessness, cough, wheeze, chest tightness, and nocturnal awakening and other clinical parameters were evaluated by the attending clinicians at each hospital and retrospectively tabulated by the authors during the study. Each symptom was scored from 0 to $3(0=$ none, $1=$ occasionally or mild, $2=$ most of the time or moderate, $3=$ all the time or severe). A patient was considered to have had symptomatic improvement if there was improvement in more than 50\% of his/ her overall total symptoms at the time of assessment by the time of assessment for the time point evaluated.

Immunological improvement was present if there was reduction in fungal serology as demonstrated by total $\operatorname{IgE}$ or radioallergosorbent test (RAST) IgE concentrations.

Radiological improvement was considered present if there was any improvement in the extent of any of the abnormalities that included bronchiectasis (severity and/or distribution), fibrosis, consolidation, mucus plugging, lobar collapse, pulmonary nodules, and/or cavitations. All image reports and images on PACS were reviewed. If any conflicting data or report was found, further review by one of two senior specialist chest radiologists was sought.

Clinical deterioration Chronic cavitory pulmonary aspergillosis (CCPA)/Chronic pulmonary aspergillosis (CPA )was considered present if intercurrent infection was absent but respiratory and constitutional signs and symptoms were progressive or there was a decline in asthma control as defined by the GINA criteria (41-43).

Radiological deterioration was considered present if there was (i) increased severity of bronchiectasis, (ii) appearance of new pulmonary nodules, cavitations, collapse, and so on, (iii) increased size and severity of existing radiological abnormalities, (iv) progressive pulmonary fibrosis, and/or (v) progression to Chronic cavitory pulmonary aspergillosis (CCPA)/Chronic pulmonary aspergillosis (CPA).

Treatment failure overall was defined by a lack of response, using the criteria above for response. Stability was defined as lack of deterioration or improvement.

Relapse after discontinuation of therapy was defined by deterioration in the same features conferring response after an initial response.

\section{Data Collection}

Clinical data from 3 to 6 months prior to the commencement of voriconazole or posaconazole (visit -1) were extracted from the clinic notes and again collected at the start of treatment (visit 0) and after 3, 6, 12, and 18 months of therapy. The data collected at visit -1 included reasons for discontinuing itraconazole, comorbidities, weight, symptoms, spirometry, and immunological markers. These data were again collected at each subsequent visit including the number of hospitalizations, GP (general practitioner) or emergency room visits, exacerbations and dose of steroids, therapeutic drug levels (TDM), adverse effects, and any new radiological findings. For the purpose of this study, GP records were not sought.
Prednisolone was used as the reference steroid when comparisons of OCS were required. Similarly, beclomethasone dipropionate (BDP) equivalent dose was used as reference for ICS. Common ICS used in our center include BDP, budesonide (1:1.5 dose equivalent), and fluticasone (1:2 dose equivalents with BDP).

Duration of therapy was calculated at the minimum time period, that is, patients who had received less than 6 months of antifungal therapy at the time of this study were evaluated at 3 months only, those who had received between 6 and 12 months were evaluated up to 6 months, and so on.

\section{Statistical Analysis}

All analyses were performed with the SPSS 15.0 software (IBM Corporation, United States of America) for Windows and Excel statistics. Descriptive statistics were used to summarize the data. To compare continuous variables before and after antifungal therapy, nonparametric Wilcoxon signed rank $T$ test for related samples was used. For all comparisons, $p$-values $\leq .05$ were considered statistically significant.

\section{RESULTS}

\section{Patient Characteristics}

There were 25 patients included in the study, $20(80 \%)$ of whom had ABPA and 5 (20\%) had SAFS (Table 1). Of all patients, $10(40 \%)$ were male and the median age was 58 years (range 20-80). ABPA patients had a higher mean age compared with SAFS (60 vs. 54 years) patients.

Itraconazole had been given to all patients for a duration of between 6 months and 10 years previously but was discontinued because of lack of efficacy $(n=11,42 \%)$ or AEs $(n=12,46 \%)$, persistently low serum concentrations $(n=2,7.8 \%)$, or itraconazole resistance in A. fumigatus $(n=2,7.8 \%)$.

Demographic variables and comorbidity prevalence are listed in Table 1.

There were 33 courses of therapy analyzed, 24 with voriconazole $(\mathrm{ABPA}=19, \mathrm{SAFS}=5)$ and 9 with posaconazole (all ABPA; Table 1). Antifungal therapy with voriconazole was modified to posaconazole in $8(32 \%)$ patients. Reasons for switch-over in these patients were side effects $(n=5)$ or lack of response $(n=3)$. Only one posaconazole course was not preceded by voriconazole due to in vitro resistance in A. fumigatus. The mean treatment duration of voriconazole was 16.7 months (range 248 months, $\mathrm{SD}=14.6)$ and of posaconazole was 16.3 months (range 3-36 months, SD =14.7). None of the SAFS patients received posaconazole (Table 1).

Of all patients, $21(80.1 \%)$ had baseline chest radiology done at the start of the therapy: chest radiography alone $(n=6,28.6 \%)$, computed tomography (CT; $n=7$, $26.9 \%)$, or both $(n=8,38 \%)$. Bronchiectasis was the most common radiological finding $(n=17,71.4 \%)$. Other common radiological findings are listed in Table 1. Two (9.5\%) of the 21 cases of ABPA analyzed had no bronchiectasis, whereas $1(20 \%)$ of the 5 cases of SAFS had mild bilateral lower lobe bronchiectasis. 
L. CHISHIMBA ET AL.

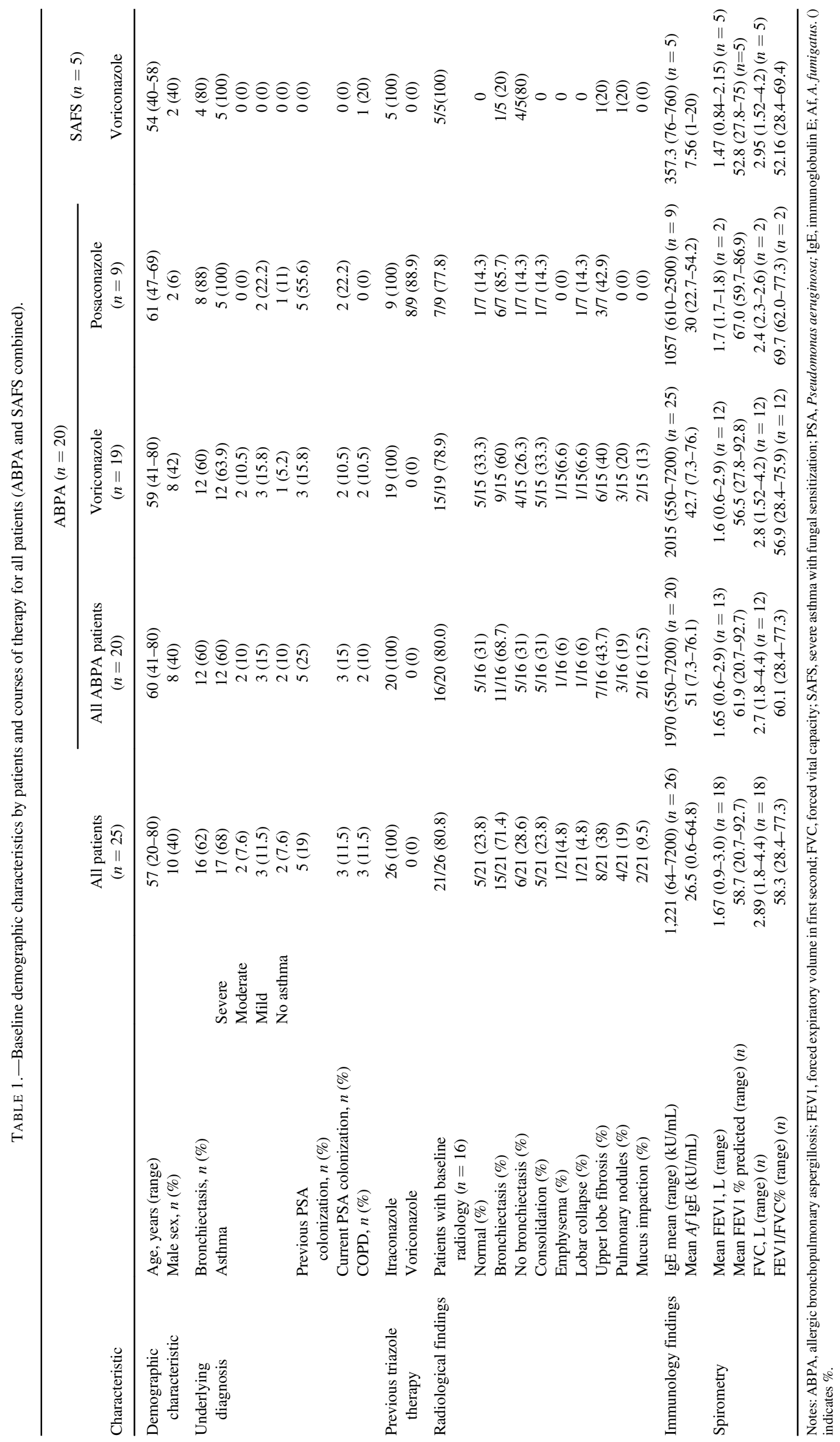




\section{Response to Therapy}

Clinical response at 3,6, and 12 months of voriconazole or posaconazole treatment is summarized in Table 2. Overall clinical improvement to voriconazole treatment was observed in $17(68 \%$; $\mathrm{ABPA}=13, \mathrm{SAFS}=4)$ of 25 patients at 3 months, $15(75 \%)$ of 20 at 6 months, and $12(70.6 \%)$ of 17 at 12 months, compared with 7 of $9(78 \%)$ at 3,6, and 12 months for posaconazole (Table 2). On the basis of clinical parameters, treatment failure to voriconazole was observed in 1 of $20(5 \%)$ patients at 3 months, none of $15(0 \%)$ at 6 months, and 2 of $15(15 \%)$ at 12 months. No treatment failure was observed with posaconazole.

There was a marked reduction in OCS and short-acting beta-2 agonist (SABA) use, health-care utilization due to asthma, and improvement in overall health status, as subjectively perceived by individual patients in domains such as physical well-being, functioning, energy, increased ET, and reduction in patients' overall symptoms (see Table 3 ). There was a direct correlation between OCS use and other clinical parameters of clinical response. Although the dosage of ICS was reportedly modified during antifungal therapy in some patients, it was difficult to quantify retrospectively, and overall was probably not markedly reduced.

There were 10 patients who had had frequent hospital admissions at the start of voriconazole therapy and no admissions were observed in 9 of them (90\%) at 3 months. This benefit was maintained at 6 and 12 months of therapy. Voriconazole therapy reduced the frequency of recurrent chest infections or acute exacerbations in 17 of $24(70 \%), 9$ of $19(47 \%)$, and 9 of $17(52.9 \%)$ patients at 3, 6, and 12 months, respectively (Table 3). Posaconazole therapy reduced the frequency of chest infections or acute exacerbations in 7 of $9(78 \%)$ patients at 3 months and throughout the 12-month period (Table 3 ).

\section{Immunological Response}

Patient median total IgE values $(\mathrm{kU} / \mathrm{mL})$ over 12 months with ABPA only are summarized in Figure 1 and Supplementary Table E1 (http://informahealthcare.com/ doi/suppl/[doinumber]). While modest falls were seen in some patients at 3 and 6 months, it was only at 9 and 12 months that sustained and statistically significant total IgE falls were seen. At 12 months, the median $\operatorname{IgE}$ decreased by $27.3 \%$ from baseline (median IgE 895, range 64-7200) to a median IgE of $475 \mathrm{kU} / \mathrm{L}$ (range 51-3100).

RAST to Aspergillus fell, but was only significant at 12 months and beyond from $23.2 \mathrm{kUa} / \mathrm{L}$ (range 0.6-294) at baseline to $17.7 \mathrm{kUa} / \mathrm{L}$ at 12 months (range 0.6-57.4; $p=.0056$; Supplementary Table E2, Figure 2). We also observed marked heterogeneity in IgE responses with a few dramatic falls, most varying erratically through the course of the therapy and some rising. There was no consistent response between $\mathrm{IgE}$ and clinical impact and so the decision to continue or discontinue therapy was based on clinical parameters (i.e., response and adverse effects). Total IgE antibody trend in ABPA by individual patient at baseline and on therapy at 3, 6, 9, and 12 months is shown in Supplement Figure E1. None of our patients received omalizumab (Xolair).

\section{Lung Function}

Overall, lung function improved throughout the treatment period. Of the eight patients with spirometry values at both baseline and 3 months, there was a nonsignificant improvement of FEV1 5.5\% from median baseline value of $1.64(p=.25)$ and FVC improved by $16.9 \%$ from a medium value of $2.67(p=.11)$. There were no positive or negative statistically significant changes or trends witnessed beyond 3 months.

\section{Radiological Response}

Of the voriconazole-treated patients who had baseline and follow-up radiology $(n=11), 5$ of $10(50 \%), 4$ of 11 (36.4\%), and 4 of $7(57.1 \%)$ patients showed improvement in radiological abnormalities including bronchiectasis (severity and/or distribution, $n=3$ ), fibrosis $(n=2)$, consolidation $(n=4)$, mucus plugging $(n=5)$, lobar collapse $(n=3)$, pulmonary nodules $(n=7)$, and/or cavitations

TABLE 2.-Overall clinical response to therapy at different times on treatment by disease groups.

\begin{tabular}{|c|c|c|c|c|}
\hline & & \multicolumn{3}{|c|}{ Clinical outcome of courses of therapy (\%) } \\
\hline & & 3 months & 6 months & 12 months \\
\hline \multicolumn{5}{|l|}{ ABPA } \\
\hline \multirow[t]{4}{*}{ Voriconazole } & Improved & $13 / 20(65)$ & $11 / 15(73)$ & $9 / 13(69)$ \\
\hline & Stable & $2 / 20(10)$ & $2 / 15(13)$ & $2 / 13(15)$ \\
\hline & Failure & $1 / 20(5)$ & $0 / 15$ & $2 / 13(15)$ \\
\hline & Discontinued (AEs) & $4 / 20(20)$ & $2 / 15(13)$ & $0 / 13$ \\
\hline \multirow[t]{4}{*}{ Posaconazole } & Improved & 7/9 (78) & 7/9 (78) & 7/9 (78) \\
\hline & Stable & $2 / 9(22)$ & $2 / 9(22)$ & $0 / 9$ \\
\hline & Failure & $0 / 9$ & $0 / 9$ & 2/9 (22) \\
\hline & Discontinued (AEs) & $0 / 9$ & $0 / 9$ & $0 / 9$ \\
\hline \multicolumn{5}{|l|}{ SAFS } \\
\hline \multirow[t]{4}{*}{ Voriconazole } & Improved & $4 / 5(80)$ & $4 / 5(80)$ & $3 / 4(75)$ \\
\hline & Stable & $1 / 5(20)$ & $1 / 5(20)$ & $1 / 5(20)$ \\
\hline & Failure & $0 / 5$ & $0 / 5$ & $0 / 5$ \\
\hline & Discontinued (AEs) & $0 / 5$ & $0 / 5$ & $0 / 5$ \\
\hline
\end{tabular}

Notes: AEs, adverse events; ABPA, allergic bronchopulmonary aspergillosis; SAFS, severe asthma with fungal sensitization. () indicates \% 
TABLE 3.- Improvements in clinical parameters during voriconazole therapy at 3, 6, and 12 months (ABPA and SAFS patients combined).

\begin{tabular}{|c|c|c|c|c|c|c|c|}
\hline \multirow[b]{2}{*}{ Clinical or health-care utilization feature } & & \multicolumn{2}{|c|}{3 months $(\%)$} & \multicolumn{2}{|c|}{6 months $(\%)$} & \multicolumn{2}{|c|}{12 months $(\%)$} \\
\hline & & $\begin{array}{c}\text { Vori } \\
(n=25)\end{array}$ & $\begin{array}{l}\text { Posa } \\
(n=9)\end{array}$ & $\begin{array}{c}\text { Vori } \\
(n=19)\end{array}$ & $\begin{array}{c}\text { Posa } \\
(n=9)\end{array}$ & $\begin{array}{c}\text { Vori } \\
(n=17)\end{array}$ & Posa $(n=9)$ \\
\hline \multirow[t]{4}{*}{ Symptoms } & Reduction in cough frequency $(\%)$ & $17 / 24(70)$ & $7 / 9(78)$ & $15 / 19(78)$ & $6 / 9(67)$ & $7 / 17(41)$ & 8/9 (89) \\
\hline & Reduction in breathlessness (\%) & $10 / 24(41)$ & $5 / 9(56)$ & $12 / 19(63)$ & 4/9 (44) & $7 / 17(41)$ & $4 / 9(44)$ \\
\hline & Increased energy $(\%)$ & $8 / 24(33)$ & $4 / 9(44)$ & $8 / 19(42)$ & 4/9 (44) & $7 / 17(41)$ & $5 / 9(56)$ \\
\hline & Reduced chest infections (\%) & $17 / 24(70)$ & $7 / 9(78)$ & $9 / 19(47)$ & $7 / 9(78)$ & $9 / 17(53)$ & $7 / 9(78)$ \\
\hline \multirow[t]{4}{*}{ Medication use } & Reduction in oral antibiotics use (\%) & $16 / 24(67)$ & $7 / 9(78)$ & $11 / 19(58)$ & $7 / 9(78)$ & $11 / 17(64)$ & 6/9 (78) \\
\hline & Reduction in OCS use (\%) & 4/18 (22) & 2/9 (29) & $5 / 18(28)$ & 2/7 (29) & $5 / 17(29)$ & 2/7 (29) \\
\hline & Discontinuation of OCS (\%) & $8 / 18(33)$ & $4 / 7(57)$ & $12 / 18(67)$ & $4 / 7(57)$ & $15 / 17(88)$ & $3 / 7(43)$ \\
\hline & Reduction in SABA use (\%) & $12 / 25(48)$ & $6 / 9(67)$ & $8 / 19(42)$ & $5 / 9(56)$ & $10 / 17(58)$ & $7 / 9(78)$ \\
\hline \multirow[t]{2}{*}{ Health-care service use } & Reduction in hospital admissions (\%) & $9 / 10(90)$ & $1 / 2(50)$ & $9 / 10(90)$ & $1 / 2(50)$ & $9 / 10(90)$ & $2 / 2(100)$ \\
\hline & Reduction in GP/emergency visits (\%) & $13 / 25(52)$ & $6 / 9(67)$ & $11 / 19(58)$ & $8 / 9(89)$ & $12 / 17(71)$ & $6 / 9(67)$ \\
\hline \multirow[t]{3}{*}{ Quality of life } & Reduction in patients' overall symptoms (\%) & $18 / 25(72)$ & $7 / 9(78)$ & $13 / 19(68)$ & $7 / 9(78)$ & $10 / 17(58)$ & $7 / 9(78)$ \\
\hline & Increased exercise tolerance (\%) & $7 / 25(28)$ & 4/9 (44) & $6 / 19(31)$ & $5 / 9(56)$ & $5 / 17(29)$ & 4/9 (44) \\
\hline & Increased QOL (\%) & $18 / 25(72)$ & $7 / 9(78)$ & $13 / 19(68)$ & $7 / 9(78)$ & $10 / 17(58)$ & $7 / 9(78)$ \\
\hline
\end{tabular}

Notes: OCS, oral corticosteroid; SABA, short-acting beta-2 agonist; QOL, quality of life; GP, general practice; ABPA, allergic bronchopulmonary aspergillosis; SAFS, severe asthma with fungal sensitization; Vori, voriconazole; posa, posaconazole. () indicates \%.

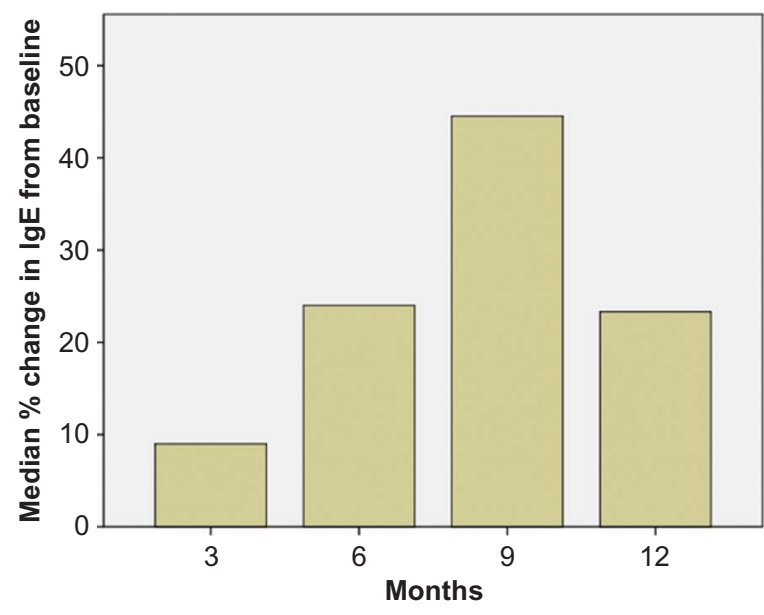

FIGURE 1.-Percentage change in median total IgE (tIgE) for ABPA and SAFS patients during 12 months of treatment with voriconazole and/or posaconazole.

Notes: At 3 months $(n=11, p=.53), 6$ months $(n=15, p=.57), 9$ months $(n=12, p=.008)$, and 12 months $(n=18, p=.025)$, respectively. IgE, immunoglobulin E; ABPA, allergic bronchopulmonary aspergillosis; SAFS, severe asthma with fungal sensitization.

$(n=4)$ at 6,12 , and 18 months of therapy, respectively. Two patients on voriconazole were noted to have worse radiological features at 3 months of therapy. Radiological deterioration was observed in 2 of $10(20 \%)$ patients at 6 months and 1 of $4(25 \%)$ patients at 12 months. Both of these cases manifested with new pulmonary nodules. One of these completely cleared after 18 months of therapy and may therefore be of uncertain significance.

One patient with ABPA developed extensive groundglass opacification (GGO) in the right upper lobe while on itraconazole (documented in vitro resistance to itraconazole and voriconazole) associated with clinical deterioration (Figure 3A). There was significant resolution in the radiological lesions except for a residual small $(17 \mathrm{~mm})$ cavitating lesion after 24 months of posaconazole therapy (Figure 3B).

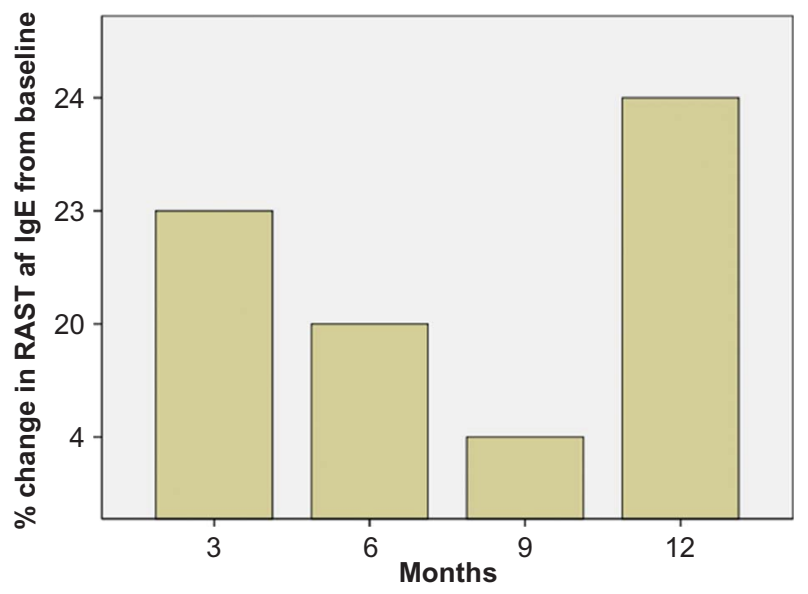

FIGURE 2.-Percentage change in median RAST IgE to af for ABPA and SAFS patients at each time point assessment during 12 months treatment with voriconazole and/or posaconazole, compared with baseline.

Notes: IgE, immunoglobulin E; ABPA, allergic bronchopulmonary aspergillosis; SAFS, severe asthma with fungal sensitization; RAST, radioallergosorbent test; af, Aspergillus fumigatus. At 3 months $(n=10, p=.17)$, 6 months $(n=15, p=.76), 9$ months $(n=12, p=.65)$, and 12 months $(n$ $=18, p=.0056$ ), respectively.

Another patient with ABPA who was intolerant to itraconazole subsequently developed right upper lobe consolidation while off antifungal therapy, which cleared completely with voriconazole therapy (Figure 4A and B).

During posaconazole therapy, radiological improvement was seen in 1 of $4(25 \%), 0$ of 4 , and 1 of $2(50 \%)$ patients' imaging at 6, 12, and 18 months of therapy, respectively. Pulmonary nodules and consolidation showed the greatest improvement, whereas fibrosis and bronchiectasis showed the least improvement.

\section{Adverse Events}

AEs occurred in $11(40 \%)$ patients taking voriconazole and $2(22 \%)$ patients taking posaconazole. Voriconazole AEs are shown in Figure 5. The most common were skin 


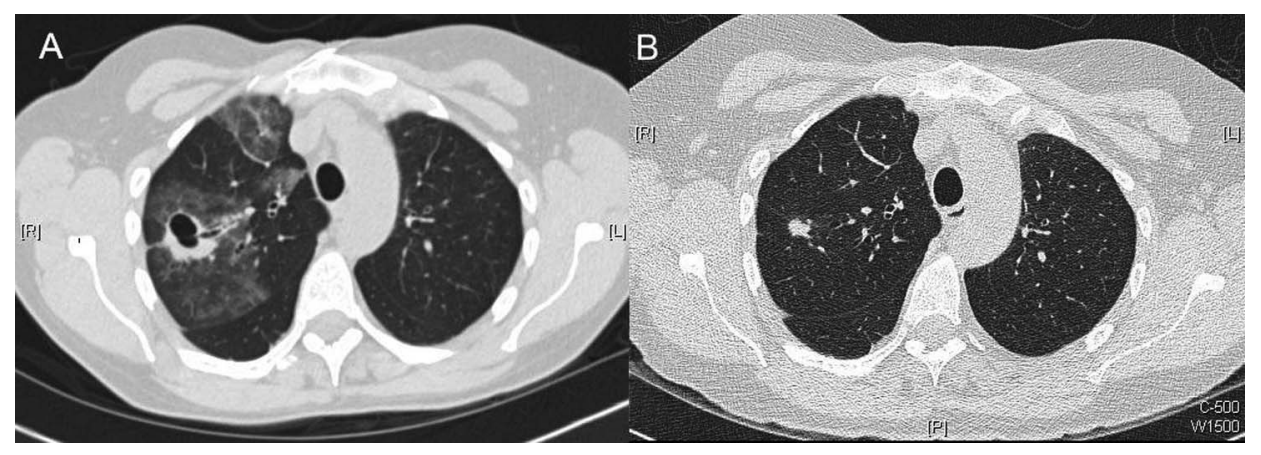

FIGURE 3.-Computed tomography (CT) chest of a 63-year-old woman with ABPA. (A) Extensive ground-glass appearances in the right upper lobe appeared while on itraconazole (documented in vitro resistance to itraconazole and voriconazole) associated with clinical deterioration (June 2008). (B) Significant resolution in the radiological lesions except for a residual small $(17 \mathrm{~mm}$ ) cavitating lesion after 24 months of posaconazole therapy (May 2010).

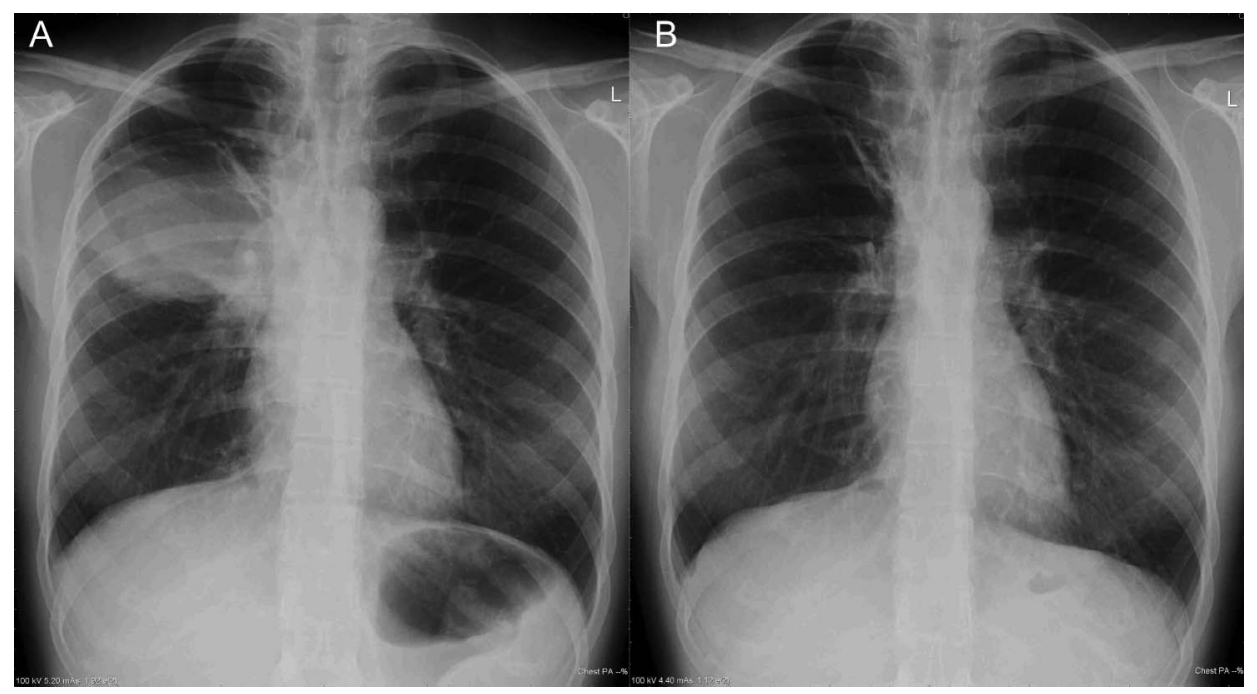

FIGURE 4.-Chest radiographs (CXR) of a 69-year-old woman with ABPA intolerant to itraconazole (after 2 months of therapy) due to adverse effects.

Notes: Patient developed right upper lobe consolidation while off antifungal therapy. She had no clinical or radiological improvement with broad spectrum antibiotics (amoxicillin + clarithromycin (1 week) followed by IV tazocin (10 days) and later IV meropenum (7 days)). Microbiological analysis from BAL samples yielded azole-sensitive A. fumigatus and negative for TB or malignancy. The patient showed good clinical improvement within 4 weeks of voriconazole therapy associated with improvement in serial CXRs. (B) The chest radiograph taken after 12 months of therapy showing complete clearance in the RUL consolidation.

photosensitivity $(n=11)$ and visual light flashes $(n=10)$. Most of these side effects were transient and mild, lasting less than a week except for some cases of photosensitivity and visual hallucinations. Significant AEs resulting in discontinuation of treatment included depression and sleep disturbance (1), photosensitivity (4), visual hallucinations (2), and peripheral neuropathy (1).

Posaconazole AEs included insomnia $(n=2)$, GI upset, and mild liver impairment $(n=1)$. No patient discontinued posaconazole because of AEs. No patient with overt Cushing's syndrome was noted during therapy with either posaconazole or voriconazole.

Two $(8 \%)$ patients on voriconazole developed adrenal suppression thought to be induced by interaction between ICS (flixotide part of seretide) and voriconazole, which has not been previously described. Subsequently, seretide was changed to serevent with ciclesonide and both patients started on regular oral hydrocortisone replacement after endocrinology review.

\section{Relapse after Discontinuation}

Among the seven patients who discontinued voriconazole, four relapsed (one at 3 months and three at 12 months).

Exclusions. We excluded one patient from the analysis. This patient had hyper-IgE syndrome (male, age 21 years) who was also sensitized to multiple fungal allergens including A. fumigatus (voriconazole group treated for 14 months) and had mild bronchiectasis on HRCT. His baseline IgE was 25,000 kU/L and he had less than two data points with respect to spirometry and radiology. His treatment was characterized by multiple disruptions due to repeated hospital admissions (ITU and general ward). He was subsequently started on azathioprine (AZA) as a steroid sparing agent to control his severe atopic asthma and eczema, which stabilized his disease activity, hence classified as a voriconazole failure. 


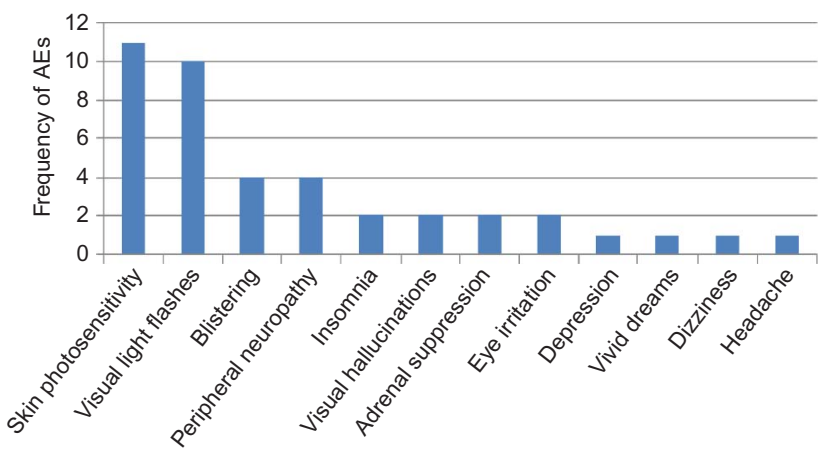

FIGURE 5.-Adverse events seen in 25 patients treated with voriconazole evaluated after $2-48$ months of treatment.

Notes: AE, adverse event. Voriconazole AEs included GI upset (7), skin photosensitivity (11), blistering (4), visual light flashes (10), insomnia (2), visual hallucinations (2), depression (1), adrenal suppression (2), peripheral neuropathy (4), eye irritation (2), vivid dreams (1), dizziness (1), and headache (1), but most of them were transient and mild lasting less than a week except for some cases of photosensitivity and visual hallucinations.

\section{DISCUSSION}

Numerous extrinsic factors are known to worsen asthma control, but the most common trigger is continuous exposure to allergens, of which fungi are important factors (44). In 2007, Bandres Gimeno and Munoz Martinez reported the first case of ABPA successfully treated with voriconazole with clinical improvement and reduction in IgE levels (45). However, there are no previously published studies to our knowledge that have directly assessed the effects of voriconazole or posaconazole therapy on asthma severity, health-care service use, and QOL in individuals with SAFS or ABPA. We performed a retrospective review of 25 patients at the North West Lung Centre (NWLC) in Manchester, UK. In our cohort, we found that voriconazole therapy reduced frequent hospital admissions in $90 \%$ of the patients at 3 months, a benefit that was maintained at 6 and 12 months of therapy. We also demonstrated a reduction in the frequency of recurrent chest infections or acute exacerbations in 17 of 24 (70\%), 9 of 19 (47\%), and 9 of $17(52.9 \%)$ patients at 3,6 , and 12 months, respectively. The observed clinical response over time to voriconazole or posaconazole is a clinically significant finding that has implications for management of patients with poorly controlled severe asthma who are allergic to fungi and support previous suggestions linking mold sensitization with severe asthma attacks requiring hospital admission (5).

\section{Asthma Control}

The current goal of asthma treatment is to achieve and maintain optimal asthma control mostly using the GINA control criteria for day- and night-time symptoms, need for rescue medication, activity limitation and exacerbations, and lung function (46, 47). Since 1999, the British Guidelines on the Management of Asthma have been produced jointly by the BTS and the Scottish Intercollegiate Guideline Network (SIGN), and achieving asthma control parameters is a key part of new versions of these guidelines (48).
In this study, we demonstrated that identification and treatment of SAFS and asthmatic ABPA patients with voriconazole and posaconazole resulted in better asthma control as demonstrated by reduction in symptoms, improvement of patients' perception of QOL, and stabilization in lung function throughout the active treatment period (Table 3). Our study supports the findings from the FAST study, a double-blind, placebo-controlled, randomized trial of itraconazole in patients with SAFS (28), and the retrospective study by Pasqualotto et al. (27).

In our patients, the magnitude of the effect of voriconazole and/or posaconazole on symptoms of asthma seen is encouraging. Better asthma control was achieved in over two-thirds of patients during 12 or more months of therapy. Using the GINA definition of asthma control $(46,47)$, a marked improvement in parameters of asthma control including reduction in medication use (SABA, OCS), emergency health-care use, and ET (27) was observed. Using the GINA criteria, we observed that at least $30 \%$ of our patients could be reclassified from uncontrolled to partly controlled asthma (47). We also observed a major reduction in cough frequency $(>67 \%)$, reduction in breathlessness $(>40 \%)$, and reduced exacerbations $(>75 \%)$ at 3 , 6 , and 12 months.

A major potential benefit of better asthma control is reduction in corticosteroid exposure. In our patients, over $30 \%$ of steroid-dependent asthmatic ABPA and SAFS patients could discontinue OCS completely with voriconazole or posaconazole therapy.

Not only are patients with severe asthma heavily burdened by disease, but the health costs for these asthma sufferers individually and to health-care services are enormous (49). There appears to be a relationship between asthma control status and urgent health care. In a recently published Asthma Insights and Reality in Asia-Pacific Phase 2 (AIRIAP 2) study, a cross-sectional, communitybased survey of 4805 subjects looking at the relationship between control status derived from the GINA and urgent health-care utilization and the relationship with selfreported urgent health-care utilization related to asthma over the previous 12 months, Lai and colleagues found that each of the symptom criteria was significantly associated with urgent health-care utilization (50). In our study, we have shown that with voriconazole or posaconazole therapy, there is significant reduction in health-care service use with reduction in hospital admissions by $90 \%$ and $50 \%$ at 3 months and $90 \%$ and $100 \%$ at 6 months, respectively. This outcome was mirrored by reduction in GP or emergency visits by $72 \%$ and $78 \%$ at 3 months, $68 \%$ and $78 \%$ at 6 months, and $58 \%$ and $78 \%$ at 12 months for voriconazole and posaconazole, respectively.

\section{Monitoring Improvement Serologically and Physiologically}

There is very little research in the current literature with regard to serological response to voriconazole in patients with ABPA or SAFS, although a retrospective review by Glackin et al. of CF patients with ABPA treated with voriconazole demonstrated a significant drop in IgE levels 
posttreatment (35). In our study, we demonstrated that treatment with voriconazole or posaconazole resulted in immunological improvement, but statistically significant reduction in immunological markers started appearing at 9 months $(p=.008)$ for total $\mathrm{IgE}$, although this was not mirrored by A. fumigatus-specific $\operatorname{IgE}$ levels until 12 months of treatment $(p=.025)$ for both total and RAST (Aspergillus-specific IgE; Figures 1 and 2 and Supplementary Tables E1 and E2). This raises an important question as to which immunological marker may be most useful for monitoring therapy and disease activity. These slow serological responses suggest that reduction in fungal burden as a cause is likely, but more rigorous means of confirming this fact are required.

A major problem with the use of total IgE concentrations for monitoring or guiding therapy is its significant variability over time reflecting the many environmental and host factors influencing its concentration. In the German Multicenter Allergy Study, assessment of correlations of total IgE levels from birth to 10 years of age in the birth cohort showed that in childhood, total IgE levels demonstrate remarkable variation over time even in the absence of atopy (51).

Another problem is that the inability to measure IgEbased sensitivity to all allergens has limited our understanding of what portion of asthma or other allergic diseases is related to IgE. In our study, we observed marked heterogeneity in $\operatorname{IgE}$ responses with a few dramatic falls, most varying erratically through the course of the therapy and some rising within the same individual with little correlation with clinical parameters, although the numbers are small and the data are not collected with precision.

Improvement or stability of lung function is one of the key objectives of asthma treatment and is one of the indices of the GINA asthma control criteria (47) and forms a major focus of asthma management by the BTS $(38,52,53)$, ATS (49), ERS, Canadian Asthma Consensus Guidelines (54, 55), and other asthma societies. We did not demonstrate a statistically significant improvement in lung function in our patients. Several other studies have demonstrated a poor relationship between measures of asthma control and lung function in patients with asthma. The relationship between FEV1, asthma severity, and symptom is poor, especially in those with fixed airway obstruction and airway remodeling (56). Our findings are similar to previous findings using itraconazole (25-27).

\section{Other Potential Long-Term Benefits of Antifungal Therapy}

There is still ongoing debate as to whether antifungal treatment could ameliorate or reverse lung destruction in allergic fungal disease. We observed in our patients that in some cases, antifungal treatment did reverse some radiological abnormalities in $25-50 \%$ patients, especially pulmonary nodular shadowing. The improvement in upper lobe cavitating mass and extensive surrounding GGO in one of our patients over 18 months of posaconazole therapy (Figure 3) and the dramatic improvement of right upper lobe consolidation while on voriconazole therapy in another with ABPA (Figure 4) are encouraging. Both patients were intolerant to itraconazole previously.

\section{Adverse Events}

Several adverse effects have been reported with voriconazole use, including acute and chronic cutaneous adverse effects, mainly due to phototoxicity. According to safety data in studies assessing voriconazole effectiveness (primarily invasive aspergillosis), $8 \%$ of outpatients experienced phototoxic events (57). More recently, some authors have reported that voriconazole was involved in the occurrence of multiple and often aggressive cutaneous squamous cell carcinomas in immunocompromised patients if the treatment was maintained for a long time (58). In our study, skin photosensitivity occurred in $11(28 \%)$ patients (Figure 5). We did not observe any skin cancers, but this possibility is worth following up.

Other voriconazole side effects in our patients were gastrointestinal upsets $(n=7)$, blistering $(n=4)$, visual light flashes $(n=10,34 \%)$, insomnia $(n=2)$, visual hallucinations $(n=2)$, depression $(n=1)$, adrenal suppression $(n=2)$, peripheral neuropathy $(n=4)$, eye irritation $(n=2)$, vivid dreams $(n=1)$, dizziness $(n=1)$, and headache $(n=1)$, but most of these were transient and mild lasting less than a week except for some cases of photosensitivity and visual hallucinations (Figure 5). We observed that five (20\%) developed significant AEs to voriconazole requiring switch-over to posaconazole.

We did not observe any significant AEs to posaconazole, although some other authors have reported a variety of AEs that appear to be unrelated to dose. In an evaluation of 18 healthy volunteers, Moton and colleagues reported the frequency of AEs when compared with placebo as headache (17\% vs. $13 \%)$, dry mouth (9\% vs. $0 \%)$, and dizziness (6\% vs. $2 \%)$, but there were no clinically significant changes in vital signs or laboratory test parameters except for transient, mild-to-moderate elevations in liver function test results (59). These authors also demonstrated a minimal impact on QT interval with posaconazole (60). Our study suggests that while voriconazole is efficacious, the possibility of serious AEs is strong, and both patient advice and close monitoring in the first few weeks of therapy are appropriate.

\section{Study Limitations}

In this study, a number of limitations exist that include small sample size, its retrospective design and subjective reporting, assessment, and reporting by different individual doctors increasing the risk of variable observer bias (60). In addition, some patients were treated with both agents and differing response parameters were necessarily used for ABPA and SAFS. Only 14 (54\%) of our patients had both baseline and follow-up imaging done; many were essentially normal at baseline. 


\section{CONCLUSIONS}

Our study shows that both voriconazole and posaconazole are potentially effective alternative treatment options for SAFS and ABPA and may improve asthma control and reduce severity. However, larger prospective studies are required, including randomized studies with good power. The possibility of AEs linked to voriconazole needs to be taken into account and needs to be monitored. There is also a need to standardize the duration of antifungal therapy, which we suggest should not be less than 6 months, and 12 months may be preferable (45). A major current limitation is drug acquisition cost, but the high rate of efficacy shows that treatment with these agents as second-line therapy is justified in certain patients.

\section{ACKNOWLEDGEMENTS}

The authors thank Mrs Julie Morris, medical statistics, University Hospital of South Manchester, Manchester, UK, for helping with statistics and Mr Paul Somerset, medical illustrations, University Hospital of South Manchester, Manchester, UK, for helping with formatting the image files.

Dr Livingstone Chishimba was involved in the conception and design of the study; acquisition, analysis, and interpretation of the data; and wrote the article. Dr Robert Niven was substantially involved in its revision prior to submission. Prof David Denning was involved in the conception and design of the study and substantial involvement in its revision prior to submission. John Cooley analyzed and interpreted the information.

\section{DECLARATION OF INTERESTS}

Dr Livingstone Chishimba has no conflict of interest to declare. Mr John Cooley has no conflicts of interest to declare. Dr Robert Niven has provided expert views on management of fungal associated asthma to Vectura as part of an advisory board. He has performed lectures at national and international education meetings supported by GSK, Novartis, Chiesi, and AZ. He has also received travel grants from Novartis, Boehringer, and GSK to attend international educational and academic meetings. Prof David Denning has received consulting fee and honorarium from Pfizer, Merck, Astellas, and Gilead. He has received support for travel to meetings from Gilead and Astellas. He also runs the Aspergillus Website (www.aspergillus.org. uk) which has been supported by Pfizer, Merck, Gilead, Astellas, and the NHS.

\section{REFERENCES}

1. Denning DW, O'Driscoll BR, Hogaboam CM, Bowyer P, Niven RM. The link between fungi and severe asthma: a summary of the evidence. Eur Respir J 2006; 27(3):615-626.

2. Porter P, Susarla SC, Polikepahad S, Qian Y, Hampton J, Kiss A, Vaidya S, Sur S, Ongeri V, Yang T, Delclos GL, Abramson S, Kheradmand F, Corry DB. Link between allergic asthma and airway mucosal infection suggested by proteinase-secreting household fungi. Mucosal Immunol 2009; 2(6):504-517.

3. Madani Y, Barlow A, Taher F. Severe asthma with fungal sensitization: a case report and review of literature. J Asthma 2010; 47(1):2-6.

4. Agarwal R, Nath A, Aggarwal AN, Gupta D, Chakrabarti A. Aspergillus hypersensitivity and allergic bronchopulmonary aspergillosis in patients with acute severe asthma in a respiratory intensive care unit in North India. Mycoses 2009; 53(2):138-143.

5. O'Driscoll BR, Hopkinson LC, Denning DW. Mold sensitization is common amongst patients with severe asthma requiring multiple hospital admissions. BMC Pulm Med 2005; 5:4.

6. Donnelly SC, McLaughlin H, Bredin CP. Period prevalence of allergic bronchopulmonary mycosis in a regional hospital outpatient population in Ireland 1985-88. Ir J Med Sci 1991; 160(9):288-290.

7. Eaton T, Garrett J, Milne D, Frankel A, Wells AU. Allergic bronchopulmonary aspergillosis in the asthma clinic. A prospective evaluation of CT in the diagnostic algorithm. Chest 2000; 118(1):66-72.

8. Patterson R, Greenberger PA, Halwig JM, Liotta JL, Roberts M. Allergic bronchopulmonary aspergillosis. Natural history and classification of early disease by serologic and roentgenographic studies. Arch Intern Med 1986; 146(5):916-918.

9. Chetty A. Pathology of allergic bronchopulmonary aspergillosis. Front Biosci 2003; 8:e110-e114.

10. Safirstein BH, D‘Souza MF, Simon G, Tai EH, Pepys J. Five-year follow-up of allergic bronchopulmonary aspergillosis. Am Rev Respir Dis 1973; 108(3):450-459.

11. Smith NL, Denning DW. Underlying conditions in chronic pulmonary aspergillosis, including simple aspergilloma. Eur Respir J. 2011 Apr;37 (4):865-872. Epub 2010 Jul 1.

12. O'Connor TM, O'Donnell A, Hurley M, Bredin CP. Allergic bronchopulmonary aspergillosis: a rare cause of pleural effusion. Respirology 2001; 6(4):361-363.

13. Fairs A, Agbetile J, Hargadon B, Bourne M, Monteiro WR, Brightling CE, Bradding P, Green RH, Mutalithas K, Desai D, Pavord ID, Wardlaw AJ, Pashley CH. IgE sensitization to Aspergillus fumigatus is associated with reduced lung function in asthma. Am J Respir Crit Care Med 2010; 182(11):1362-1368.

14. Greenberger PA. Diagnosis and management of allergic bronchopulmonary aspergillosis. Allergy Proc 1994; 15(6):335-339.

15. Imbeau SA, Nichols D, Flaherty D, Dickie H, Reed C. Relationships between prednisone therapy, disease activity, and the total serum IgE level in allergic bronchopulmonary aspergillosis. J Allergy Clin Immunol 1978; 62(2):91-95.

16. Clayton DE, Busse WW. Development of allergic bronchopulmonary aspergillosis during treatment of severe asthma with systemic corticosteroids. J Allergy Clin Immunol 1981; 67(3):243-246.

17. Laufer P. Assessment of corticosteroid therapy for allergic bronchopulmonary aspergillosis in a patient with cystic fibrosis. J Asthma 1985; 22(5):253-255.

18. Zhang DH, Li HD, Zheng L. [Diagnosis and treatment of allergic bronchopulmonary aspergillosis]. Zhonghua Nei Ke Za Zhi 1987; 26(1):6-8.

19. Heinig JH, Weeke ER, Groth S, Schwartz B. High-dose local steroid treatment in bronchopulmonary aspergillosis. A pilot study. Allergy 1988; 43(1):24-31.

20. Denning DW, Van Wye JE, Lewiston NJ, Stevens DA. Adjunctive therapy of allergic bronchopulmonary aspergillosis with itraconazole. Chest 1991; 100(3):813-819.

21. Fujimori Y, Tada S, Kataoka M, Kawaraya M, Ikubo S, Horiba M, Okahara M, Takehara H, Harada M, Tanabe K. Allergic bronchopulmonary aspergillosis effectively treated with itraconazole. Nihon Kokyuki Gakkai Zasshi 1998; 36(9):781-786.

22. Nikaido Y, Nagata N, Yamamoto T, Yoshii C, Ohmori H, Kido M. A case of allergic bronchopulmonary aspergillosis successfully treated with itraconazole. Respir Med 1998; 92(1):118-119.

23. Leon EE, Craig TJ. Antifungals in the treatment of allergic bronchopulmonary aspergillosis. Ann Allergy Asthma Immunol 1999; 82(6):511516, quiz 516-519.

24. Fink JN. Therapy of allergic bronchopulmonary aspergillosis. Indian J Chest Dis Allied Sci 2000; 42(4):221-224. 
25. Wark P. Pathogenesis of allergic bronchopulmonary aspergillosis and an evidence-based review of azoles in treatment. Respir Med 2004; 98(10):915-923.

26. Wark PA, Gibson PG, Wilson AJ. Azoles for allergic bronchopulmonary aspergillosis associated with asthma. Cochrane Database Syst Rev 2003; 3:CD001108.

27. Pasqualotto AC, Powell G, Niven R, Denning DW. The effects of antifungal therapy on severe asthma with fungal sensitization and allergic bronchopulmonary aspergillosis. Respirology 2009; 14(8):11211127.

28. Denning DW, O'Driscoll BR, Powell G, Chew F, Atherton GT, Vyas A, Miles J, Morris J, Niven RM. Randomized controlled trial of oral antifungal treatment for severe asthma with fungal sensitization: the Fungal Asthma Sensitization Trial (FAST) study. Am J Respir Crit Care Med 2009; 179(1):11-18.

29. Tucker RM, Haq Y, Denning DW, Stevens DA. Adverse events associated with itraconazole in 189 patients on chronic therapy. J Antimicrob Chemother 1990; 26(4):561-566.

30. Lestner JM, Roberts SA, Moore CB, Howard SJ, Denning DW, Hope WW. Toxicodynamics of itraconazole: implications for therapeutic drug monitoring. Clin Infect Dis 2009; 49(6):928-930.

31. Baxter CG, Marshall A, Roberts M, Felton TW, Denning DW. Peripheral neuropathy in patients on long-term triazole antifungal therapy. J Antimicrob Chemother 2011; 66(9):2136-2139.

32. Skov M, Main KM, Sillesen IB, Müller J, Koch C, Lanng S. Iatrogenic adrenal insufficiency as a side-effect of combined treatment of itraconazole and budesonide. Eur Respir J 2002; 20(1):127-133.

33. Slordal L, Spigset O. Heart failure induced by non-cardiac drugs. Drug Saf 2006; 29(7):567-586.

34. Hilliard T, Edwards S, Buchdahl R, Francis J, Rosenthal M, BalfourLynn I, Bush A, Davies J. Voriconazole therapy in children with cystic fibrosis. J Cyst Fibros 2005; 4(4):215-220.

35. Glackin L, Leen G, Elnazir B, Greally P. Voriconazole in the treatment of allergic bronchopulmonary aspergillosis in cystic fibrosis. Ir Med J 2009; 102(1):29.

36. Ricketti AJ, Greenberger PA, Mintzer RA, Patterson R. Allergic bronchopulmonary aspergillosis. Arch Intern Med 1983; 143(8):1553-1557.

37. Patterson R, Greenberger PA, Harris KE. Allergic bronchopulmonary aspergillosis. Chest 2000; 118(1):7-8.

38. Clancy K. British guidelines on the management of asthma. Thorax 2004; 59(1):81-82.

39. Andes D, Pascual A, Marchetti O. Antifungal therapeutic drug monitoring: established and emerging indications. Antimicrob Agents Chemother 2009; 53(1):24-34.

40. Felton TW, Baxter C, Moore CB, Roberts SA, Hope WW, Denning DW Efficacy and safety of posaconazole for chronic pulmonary aspergillosis. Clin Infect Dis 2010; 51(12):1383-1391.

41. Cockcroft DW, Swystun VA. Asthma control versus asthma severity. J Allergy Clin Immunol 1996; 98(6 Pt 1):1016-1018.

42. Zervas E, Oikonomidou E, Kainis E, Kokkala M, Petroheilou K, Gaga M. Control of asthma. Ther Adv Respir Dis 2008; 2(3):141-148.

43. Bateman ED, Hurd SS, Barnes PJ, Bousquet J, Drazen JM, FitzGerald M, Gibson P, Ohta K, O'Byrne P, Pedersen SE, Pizzichini E, Sullivan SD, Wenzel SE, Zar HJ. Global strategy for asthma management and prevention: GINA executive summary. Eur Respir J 2008; 31(1):143-178.
44. Agarwal R, Gupta D. Severe asthma and fungi: current evidence. Med Mycol. 2011 Apr;49 Suppl 1:S150-7. Epub 2010 Jul 22.

45. Bandres Gimeno R, Munoz Martinez MJ. Prolonged therapeutic response to voriconazole in a case of allergic bronchopulmonary aspergillosis. Arch Bronconeumol 2007; 43(1):49-51.

46. Soto-Quiros ME. Practical handling of the asthma based on the control of the disease: update of GINA guidelines. An Pediatr (Barc) 2008; 68(4):317-319.

47. Koshak EA. Classification of asthma according to revised 2006 GINA: evolution from severity to control. Ann Thorac Med 2007; 2(2):45-46.

48. Turner S, Paton J, Higgins B, Douglas G; British Guidelines on the Management of Asthma. British guidelines on the management of asthma: what's new for 2011? Thorax 2011; 66(12):1104-1105.

49. Wenzel S. Severe asthma in adults. Am J Respir Crit Care Med 2005; 172(2):149-160.

50. Lai CK, Ko FW, Bhome A, DE Guia TS, Wong GW, Zainudin BM, Nang AN, Boonsawat W, Cho SH, Gunasekera KD, Hong JG, Hsu JY, Viet NN, Yunus F, Mukhopadhyay A. Relationship between asthma control status, the asthma control test and urgent health-care utilization in Asia. Respirology 2011; 16(4):688-697.

51. Nickel R, Illi S, Lau S, Sommerfeld C, Bergmann R, Kamin W, Forster J, Schuster A, Niggemann B, Wahn U; German Multicenter Allergy Study Group (MAS-90). Variability of total serum immunoglobulin E levels from birth to the age of 10 years. A prospective evaluation in a large birth cohort (German Multicenter Allergy Study). Clin Exp Allergy 2005; 35(5):619-623.

52. British Thoracic Society, Scottish Intercollegiate Guidelines Network. British Guideline on the Management of Asthma, A national clinical guideline,www.sign.ac.uk, Thorax. 2008 May;63 Suppl 4:iv1-121.

53. Levy ML, Thomas M, Small I, Pearce L, Pinnock H, Stephenson P. Summary of the 2008 BTS/SIGN British guideline on the management of asthma. Prim Care Respir J 2009; 18(Suppl. 1):S1-S16.

54. Boulet LP, Becker A, Bérubé D, Ernst P, Beveridge R. 1998 revision of the Canadian asthma consensus guidelines. asthma consensus conference editorial committee. Can Respir J 1999; 6(3):231-232.

55. Boulet LP, Becker A, Bérubé D, Beveridge R, Ernst P. Canadian asthma consensus report, 1999. Canadian asthma consensus group. CMAJ 1999; 161(11 Suppl.):S1-S61.

56. Pascual RM, Peters SP. Airway remodeling contributes to the progressive loss of lung function in asthma: an overview. J Allergy Clin Immunol 2005; 116(3):477-486, quiz 487.

57. Epaulard O, Leccia MT, Blanche S, Chosidow O, Mamzer-Bruneel MF, Ravaud P, Thiebaut A, Villier C, Lortholary O. Phototoxicity and photocarcinogenesis associated with voriconazole. Med Mal Infect 2011; 41 (12):639-645.

58. Cowen EW, Nguyen JC, Miller DD, McShane D, Arron ST, Prose NS, Turner ML, Fox LP. Chronic phototoxicity and aggressive squamous cell carcinoma of the skin in children and adults during treatment with voriconazole. J Am Acad Dermatol 2010; 62(1):31-37.

59. Moton A, Krishna G, Wang Z. Tolerability and safety profile of posaconazole: evaluation of 18 controlled studies in healthy volunteers. J Clin Pharm Ther 2009; 34(3):301-311.

60. Juniper EF, Chauhan A, Neville E, Chatterjee A, Svensson K, Mörk AC, Ståhl E. Clinicians tend to overestimate improvements in asthma control: an unexpected observation. Prim Care Respir J 2004; 13(4):181-184. 